\title{
EVIDENCE OF ELECTRON-PHONON INTERACTION IN THE INFRARED SPECTRA OF OXIDE SUPERCONDUCTORS
}

\author{
T. Timusk \\ McMaster University, Hamilton, Ontario, Canada L8S 4M1 \\ D.B. Tanner \\ University of Florida, Gainesville, Florida 32611 \\ ABSTRACT
}

\begin{abstract}
We show that the structure in $a b$-plane infrared spectra of the oxide superconductors can be understood in terms of the electron-phonon interaction. The minima in the optical conductivity seen in the oxygen breathing mode region translate to peaks in the frequency dependent scattering rate. The position and the width of the peaks are in reasonable agreement with structure seen in timeof-flight neutron spectroscopy. We interpret the appearance of phonon modes in the highly metallic $a b$-plane in terms of a charged phonon model borrowed from the theory of organic conductors.
\end{abstract}

\section{INTRODUCTION}

The role of phonons in the high $T_{c}$ superconductors is subtle. On the one hand there is evidence that the charge carriers responsible for superconductivity are weakly coupled to the lattice. For example, the isotope shift in in $\mathrm{YBa}_{2} \mathrm{Cu}_{3} \mathrm{O}_{7}$ is small if not zero and calculations based on the electron-phonon mechanism, while quite good at predicting $T_{c}$ 's in conventional superconductors, do not yield the high transition temperatures actually observed in the oxides. ${ }^{1}$ The temperature dependence of the resistivity is not what one expect: of phonons. The oxygen breathing modes at $500 \mathrm{~cm}^{-1}(62 \mathrm{meV})$ should givt rise to a positive curvature to the resistivity at room temperature instead o the straight line that is actually observed. The magnitude of the scatterin! rate, $1 / \tau \approx(1-2) k_{B} T$, suggests weak scattering of the carriers from som low frequency excitation, below typical phonon frequencies. Thus transpor measurements point to a weak interaction between the dc current carriers an phonons.

However spectroscopic measurements on the phonons themselves show the certain phonons are in strong contact with the electrons. Neutron time-of-fligt (TOF) spectra, ${ }^{2}$ show pronounced softening in the $50 \mathrm{meV}$ region as the mater als are changed from insulating parent compound to conductor/superconducts by oxygen doping. Fig. $1 \mathrm{a}$ and and $1 \mathrm{~b}$, from Renker et al. ${ }^{2}$ illustrate the: effects in $\mathrm{YBa}_{2} \mathrm{Cu}_{3} \mathrm{O}_{7-\delta}$. Phonon branches - related to $\mathrm{Cu}-\mathrm{O}$ stretches-shc anomalies near the zone boundary. ${ }^{3}$ Raman spectroscopy too shows that certa optic phonon frequencies shift as the materials become superconducting ${ }^{4-7}$ ar the lines show asymmetric shapes that suggest interaction of the Raman-acti modes with an underlying electronic continuum. ${ }^{8}$ This continuum gives rise tc broad band of scattering at low temperature that peaks in the $350 \mathrm{~cm}^{-1}$ regi in $\mathrm{A}_{1 g}$ symmetry. 


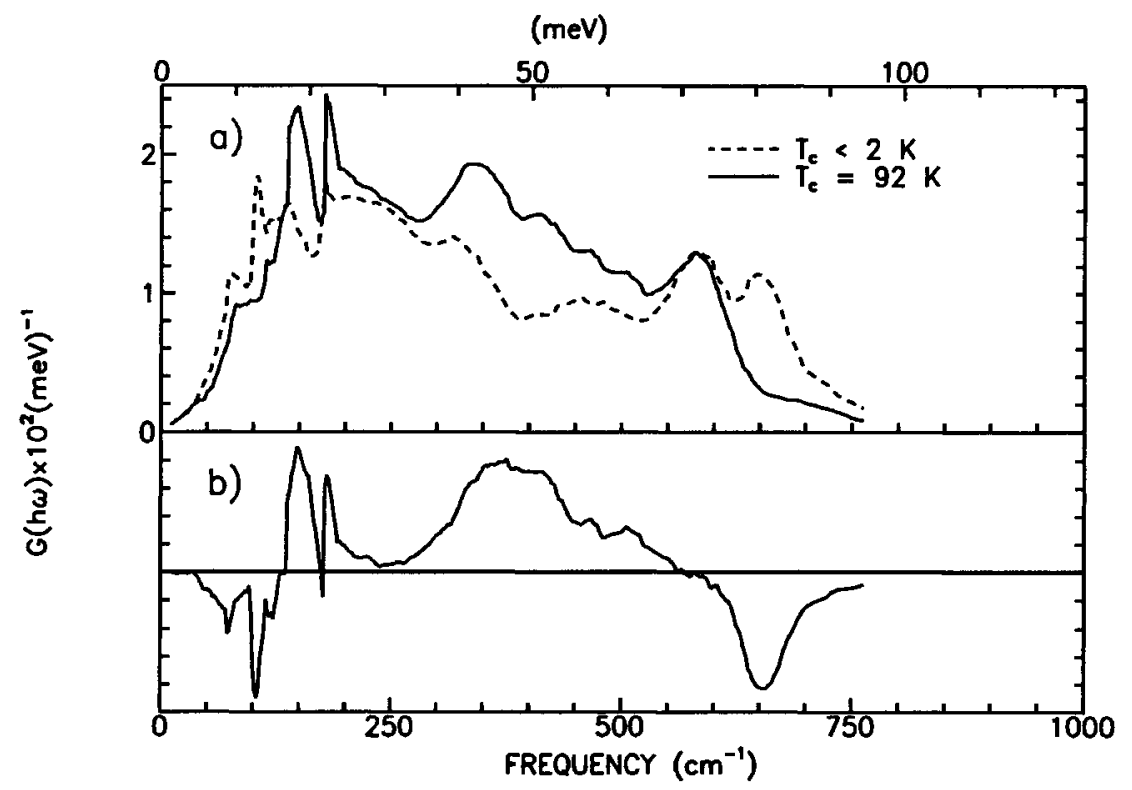

Fig. 1 a) Neutron density of states for phonons from Renker et $a l^{2}$ for $\mathrm{YBa}_{2} \mathrm{Cu}_{3} \mathrm{O}_{x}$. The solid curve is for a superconducting sample $(x=7)$ the dashed one for a non-superconducting one $(x=6)$. There is a strong enhancement of density of states in the 20 and the $50 \mathrm{meV}$ regions. b) A difference spectrum between the two curves in a).

Here we are concerned with the influence of phonons on the infrared properties of the high $T_{c}$ materials. Early work on ceramic samples exhibited a rich spectrum dominated by phonons characteristic of perovskites. ${ }^{9}$ These phonon lines were shown by Collins et al. to originate from the $c$-axis response of the crystallites. ${ }^{10}$ There are no obvious signatures of phonons in the crystal or thin film spectra for the $a b$-plane reflectance. This is not surprising since the materials are good metals and the charge carriers, not the phonons, are expected to dominate the electromagnetic response.

It is therefore surprising to see in $\mathrm{YBa}_{2} \mathrm{Cu}_{3} \mathrm{O}_{7}$, at low temperature, $a b$ plane reflectance features in the form of two "knees", one at $155 \mathrm{~cm}^{-1}(20 \mathrm{meV})$ and the other, more prominent one, at $420 \mathrm{~cm}^{-1}(52 \mathrm{meV})$ in the phonon region of the spectrum. These features exhibit characteristics of phonons: their frequency does not depend on changes in the electronic structure associated with oxygen doping ${ }^{11}$ and they do not undergo dramatic changes when the materials become superconducting, and they are present in the normal state. ${ }^{12}$ We have suggested that these features can be understood in terms of a model where the phonons acquire electronic oscillator strength by the "charged" phonon mechanism, a phenomenon seen in organic conductors, where charge density waves can give internal molecular vibrations electronic-strength infrared intensities. ${ }^{13,14}$ 


\section{PHONONS IN METALS}

Phonons in metals can absorb electromagnetic energy in several different ways. The simplest is a direct coupling of the ionic charges to the external field, the familiar reststrahlen absorption. It is expected to be weak in the oxides. Tajima et $a l^{15}$ have recently measured the oscillator strength of the $a b$-plane infrared active phonon transitions for a variety of insulating parent compounds of the high $T_{c}$ superconductors. They find rather small effective charges for the oxygens, with $Z_{O}$ ranging between 0.87 and 1.45 electronic charges. Oxygen, being the light ions of the structure, would dominate the absorption.

It is easy to estimate the strength of phonon lines expected in the superconductor. Tajima et al. find that in $\mathrm{La}_{2} \mathrm{CuO}_{4}$ a phonon has for example $\omega_{T O}=140 \mathrm{~cm}^{-1}, \omega_{L O}=320 \mathrm{~cm}^{-1}, \gamma=42 \mathrm{~cm}^{-1}$. With parameters that fit typical of good crystals in the $a b$-plane, ${ }^{12}$ a phonon of this strength at $155 \mathrm{~cm}^{-1}$ would only give a $0.01 \%$ feature in reflectance. This kind of weak structure is well below what can be seen experimentally. Clearly optical phonons are diffcult to observe in superconductors, even with an electronic background of the type seen in the high $T_{c}$ materials. This simple direct mechanism cannot be responsible for the observed phonon features at $155 \mathrm{~cm}^{-1}$ and at $360 \mathrm{~cm}^{-1}$.

In ordinary metals with strong electron-phonon interaction the Holstein ${ }^{16}$ mechanism provides observable phonon structure in the infrared. ${ }^{17}$ Each phonon branch gives rise to a threshold in absorption, above this threshold the incoming photon energy is divided between the phonon and electron-hole pair in the final state. In the superconducting state this threshold becomes a step at a frequency that is the sum of the phonon frequency and the energy necessary to break the Cooper pair $2 \Delta$. This predicted $2 \Delta$ shift at the superconducting transition of the absorption threshold of the phonons has not been observed for any feature in the spectra of high $T_{c}$ superconductors. Model calculations ${ }^{18-20}$ show that for a strong coupling Eliashberg model with a gap of $4 k_{B} T_{c}$, coupled to oxygen breathing modes, a strong threshold appears at approximately $8 k_{B} T_{c}$ in the normal state, shifting to $10 k_{B} T_{c}$ as superconductivity sets in. The absence of this $2 \Delta$ shift rules out the Holstein mechanism as the cause of the phonon features.

A mechanism that enhances the oscillator strength of optic phonons and breaks down selection rules making totally symmetrical phonons optically active is the "charged phonon" mechanism of Rice, ${ }^{13,21,22}$ known to occur in organic conductors. Associated with low-lying electronic states the symmetric lattice vibrations pump charge over large distances giving rise to absorption at phonon frequencies that is of electronic strength. The characteristic signature of this process is the appearance of sharp peaks where the background electronic absorption has gaps and sharp notches or antiresonances where the electronic background has non-zero oscillator strength. 


\section{THE OPTICAL CONDUCTIVITY AND SCATTERING RATE}

The best evidence for the charged phonon mechanism in the high $T_{c}$ superconductors comes from an analysis of the optical conductivity in the superconducting state where the Drude conductivity has collapsed to form a delta function at zero frequency, better revealing the electronic background. Fig. 2a shows the conductivity from the work of Kamarás et al. for $\mathrm{YBa}_{2} \mathrm{Cu}_{3} \mathrm{O}_{7-\delta}$. The overall amplitude of the conductivity varies between 1000 and $2500 \Omega^{-1} \mathrm{~cm}^{-1}$. The conductivity is dominated by a broad continuum peaking at $900 \mathrm{~cm}^{-1}$ with a pronounced minimum in the $500 \mathrm{~cm}^{-1}$ region. There is a a gap-like onset in the $130 \mathrm{~cm}^{-1}$ region. Other investigators generally find similar results $23,24,11,10$ although there is some variation in the details of the spectra. The gap in conductivity results from the region of unity reflectance below $130 \mathrm{~cm}^{-1}$. While accurate measurements of absolute reflectance at low frequency are difficult several groups have reported, to within an experimental accuracy of $1 \%$ or so, unity reflectance below this frequency. Structure is always observed at $155 \mathrm{~cm}^{-1}$ but not all experiments show a gap. For example recent bolometric experiments by Pham $e t$ al. ${ }^{25}$ show a a small peak at this frequency superimposed on an absorption with a quadratic frequency dependence suggestive of a finite conductivity to the lowest frequencies. A gap has also been reported by Reedyk et al. ${ }^{26}$ in $\mathrm{Bi}_{2} \mathrm{Sr}_{2} \mathrm{CaCu}_{2} \mathrm{O}_{8}$ at $300 \mathrm{~cm}^{-1}$ and like the gap in $\mathrm{YBa}_{2} \mathrm{Cu}_{3} \mathrm{O}_{7}$ this feature is present in the normal state and likely associated with phonons too.

We next calculate the frequency dependent scattering rate $\Gamma(\omega)$ associated with the conductivity in Fig. 2a. We assume that the dielectric response is made up of three parts, the Drude part $\epsilon_{D}$, a midinfrared electronic part $\epsilon_{e}$ and the high frequency contribution $\epsilon_{\infty}$ :

$$
\epsilon(\omega)=\epsilon_{D}+\epsilon_{e}+\epsilon_{\infty}
$$

Since $\epsilon_{e}$ has all the structure at low frequency ${ }^{27}$ it is helpful to write $\epsilon_{e}$ in terms of the frequency dependent scattering rate: ${ }^{28,29}$

$$
\epsilon_{e}=\frac{\omega_{p e}^{2}}{\omega I(\omega)-\omega^{2}-i \omega \Gamma(\omega)}
$$

where $\omega_{p e}$ is the plasma frequency of the electronic midinfrared band and $\Gamma(\omega)$ and $I(\omega)$ describe the real and imaginary parts of the frequency dependent scattering rate. With $\epsilon_{e}=\epsilon_{e 1}+i \epsilon_{e 2}$, we can get $\epsilon_{e 2}$ by Kramers-Kronig analysis from reflectance experiments and $\epsilon_{e 1}$ from a second Kramers-Kronig transformation of $\epsilon_{e 2}$. Thus, the frequency dependent scattering rate,

$$
\Gamma(\omega)=\frac{\omega_{p e}^{2} \epsilon_{e 2}}{\omega\left(\epsilon_{e 1}^{2}+\epsilon_{e 2}^{2}\right)}
$$

can be obtained from the experimentally determined $\sigma(\omega)$ at low temperature. The only fitted parameter used is the plasma frequency of the midinfrared band, 


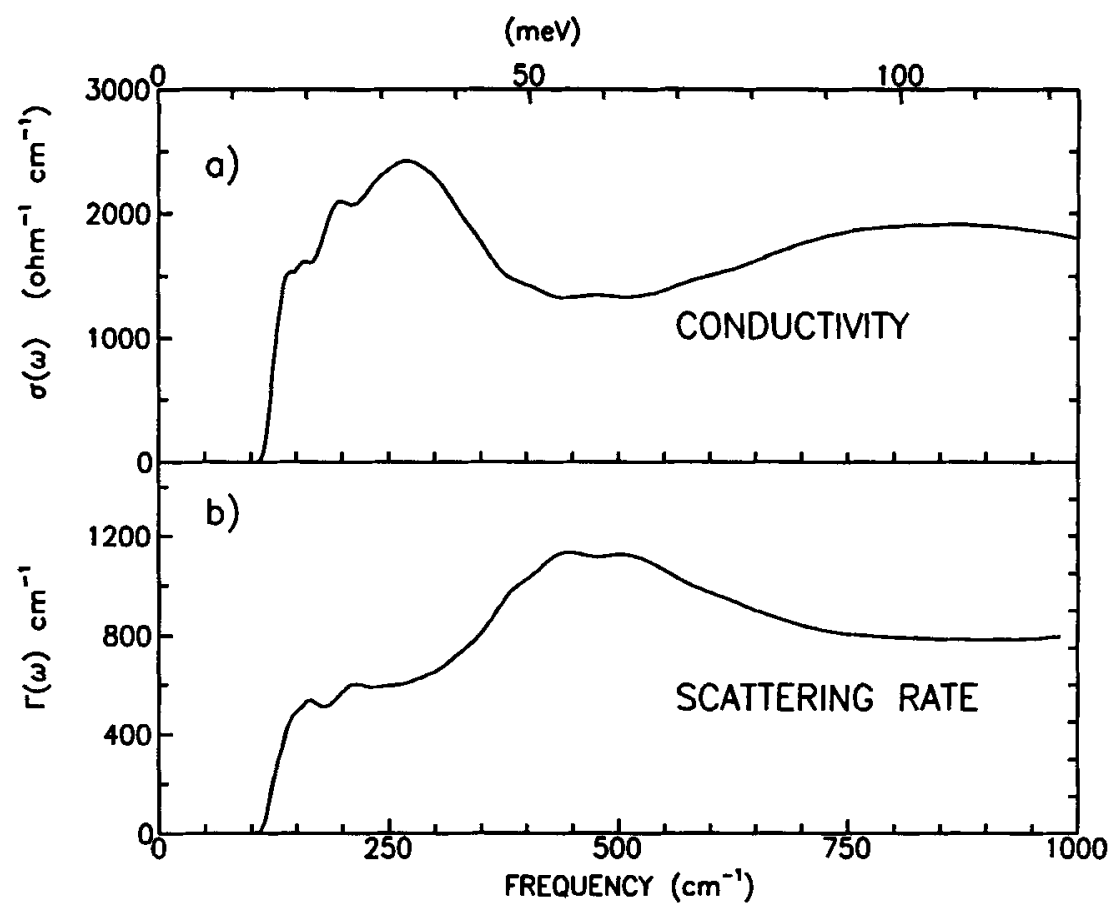

Fig. 2 a) Frequency dependent conductivity of $\mathrm{YBa}_{2} \mathrm{Cu}_{3} \mathrm{O}_{7}$ from Kamarás et al. . The spectrum is characterized by a notch-like minimum in the $450 \mathrm{~cm}^{-1}$ region and a threshold at $130 \mathrm{~cm}^{-1}$. We associate both these features with phonons. b) The frequency dependent scattering rate $\Gamma(\omega)$ obtained from the conductivity be Kramers Kronig analysis. There is a peak at the frequency of the notch in the conductivity. We attribute this peak to the same phonon branch that causes the anomalous neutron scattering seen in Fig. $1 \mathrm{~b}$.

$\omega_{p e}$ (taken from oscillator fits by Kamarás et al. ${ }^{12}$ ), which sets the overall scale of $\Gamma(\omega)$.

Fig. $2 b$ shows $\Gamma(\omega)$ obtained from the low temperature conductivity spectra of Kamarás et al. for a laser ablated film of $\mathrm{YBa}_{2} \mathrm{Cu}_{3} \mathrm{O}_{7}$. The spectrum has an onset in the $130 \mathrm{~cm}^{-1}(16 \mathrm{meV})$ region, a broad peak centered at 450 $\mathrm{cm}^{-1}(55 \mathrm{meV})$, followed by a fairly constant scattering rate of the order of 1000 $\mathrm{cm}^{-1}$. We will focus on the structure in the phonon region, $100-700 \mathrm{~cm}^{-1}$.

The most striking feature of the $\Gamma(\omega)$ spectrum is a peak in the scattering rate just below the oxygen breathing mode region. The observation of a peak rather than a threshold is clear evidence that the electron-phonon interaction here is not of the Holstein type which yields onsets but of a type where the final state only has a phonon, i.e. a charged phonon process described by Rice ${ }^{13}$. The center frequency of the peak is good agreement with the phonon band found by Renker et al. (Fig. 1b) in $\mathrm{YBa}_{2} \mathrm{Cu}_{3} \mathrm{O}_{7}$ that appeared when to doping 
level was altered to change the material from the non-superconducting $\mathrm{O}_{6}$ to the superconducting concentration $\mathrm{O}_{7}$.

The strength of the band can be described in terms of $\lambda$ the electronphonon coupling constant. We can make a rough approximation by setting $\lambda \approx \pi \Gamma\left(\omega_{0}\right) d \Gamma / 2 \omega_{0}^{2}$, where $d \Gamma$ is the width of the peak in $\Gamma\left(\omega_{0}\right)$ and $\omega_{0}$ its center frequency. With a value of $\Gamma\left(\omega_{0}\right)=1000 \mathrm{~cm}^{-1}$ a $\lambda$ of approximately 2 is found for the $450 \mathrm{~cm}^{-1}$ band. Thus the broad phonon band centered at 450 $\mathrm{cm}^{-1}$ is strongly coupled to the electronic continuum.

The onset of scattering in the $130 \mathrm{~cm}^{-1}(16 \mathrm{meV})$ region in Fig. $1 \mathrm{c}$ is a reflection of the gap-like depression in conductivity below this frequency in the spectra of Kamarás et al. At low frequency the accuracy of the experimental reflectance that has been used as input to the Kramers Kronig analysis becomes worse. We have therefore also used a model calculation to analyze the data. ${ }^{14}$ Approximating the frequency dependent scattering rate by Lorentz oscillators plus a constant term to describe the background damping, the model describes the experimental reflectance quite well with only two oscillators chosen match the neutron TOF difference peaks of Fig. 1b. In this model the lower knee is produced by the sharp $150 \mathrm{~cm}^{-1}$ double feature in the TOF spectrum and the upper one by the broader peak at $400 \mathrm{~cm}^{-1}(50 \mathrm{meV})$. The conclusion that we reach from our model calculation is that the onset and the shoulder in $\Gamma(\omega)$ at $150 \mathrm{~cm}^{-1}$ that we obtain by Kramers Kronig analysis of the existing experiments may not be correct. Instead $\Gamma(\omega)$ may have a peak at $150 \mathrm{~cm}^{-1}$ superimposed on a background. More accurate experiments are needed to settle the nature of the scattering at low frequency.

Fig. 2 demonstrates that the conductivity and scattering rate in the superconducting state of $\mathrm{YBa}_{2} \mathrm{Cu}_{3} \mathrm{O}_{7}$ has the experimental signatures of charged phonons. In reflectance optic phonons generally give rise to reflectance minima when there is a free carrier background whereas charged phonons in the presence of an electronic continuum give rise to peaks. The opposite holds for the optical conductivity. We find from our model that when the charged phonon coupling gets to be strong which is the case here, particularly in the superconducting state, the reflectance peaks turn into thresholds.

\section{DISCUSSION}

The charged phonon mechanism is well studied in organic conductors where $\mathrm{A}_{1 g}$ symmetry internal vibrations of the molecules are coupled by symmetry breaking charge density waves to the external electromagnetic field. In the case of the high- $T_{c}$ oxides, a potential will also be needed to allow IR activity of modes that are not at $q=0$, in particular the branch at the zone boundary modes seen by neutron spectroscopy to couple strongly to the electrons. Since it appears from the temperature dependent spectra of Kamarás et al. that the depth of the notch in the conductivity increases as the temperature is lowered, that is the coupling between the electrons and the zone boundary branch grows at low temperature, an incipient structural instability may generate the symmetry breaking potential. One cannot rule out the role of defects in the activation of the phonons since there is quite a large amount of sample to sample variation 
in the infrared spectra in the phonon region. ${ }^{30}$ The high degree of variability of the $155 \mathrm{~cm}^{-1}$ mode in particular suggests that its activation may be associated with defects.

In our picture of the frequency dependent conductivity, the phonons interact with the electronic continuum producing, by a Fano interference between the discrete and continuous states, the characteristic $\mathrm{v}$ shaped minima. This electronic absorption band, we argue, should be distinguished from the Drude free carrier band as a separate parallel channel of conductivity. Our analysis of the frequency dependent scattering of the part of the conductivity should be distinguished from models where the entire infrared conductivity, Drude plus midinfrared, is analyzed according to Eqn. $2 .{ }^{23,10}$

The idea of two parallel contributions to the low frequency $\sigma(\omega)$ was proposed by Schützmann et al. ${ }^{24}$ The two contributions come from the two $a b$-plane conduction bands that occur in the electronic band structure of $\mathrm{YBa}_{2} \mathrm{Cu}_{3} \mathrm{O}_{7}$. The two bands arise because there are two copper oxide layers per unit cell and these are coupled by a transverse hopping integral $t_{\perp}$. This coupling splits the degeneracy of the plane orbitals by $\pm t_{\perp}$. In the picture proposed by Schützmann et al. these two bands have different effective masses and scattering rates: both contribute to the dc conductivity. In contrast, we view one band (the Drude carriers) as being weakly coupled or uncoupled to the phonons and determining the dc conductivity. The other band couples strongly as described above. This coupling reduces the dc conductivity of the carriers in this band to zero, gives in only modest temperature dependence, and leads to absorption throughout the phonon region of the spectrum. The reason for the different behavior of these two bands we are unable to specify, but it must in some way be related to the different symmetry of the two bands (one even and the other odd under inversion, for example).

The experiments of of Kamarás et al. ${ }^{12}$ that our analysis leading to Fig. $2 b$ is based on were done on high quality twinned films and thus represent $a$ superposition of $a$ - and $b$-axis conductivities. There is evidence that the chains make a separate contribution to the conductivity in the form of a peak at 0.5 $\mathrm{eV}^{31-35}$ and recent work by Schlesinger et al. ${ }^{35}$ shows that the low frequency tail of this absorption extends down into the phonon region analyzed in this work. To account for the chains one should subtract this contribution from the midinfrared conductivity. This is difficult since the $a b$-plane itself is quite anisotropic, both because of anisotropic bond-lengths and interactions with the chain layer below.

Raman spectroscopy provides an independent confirmation of the anisotropy of the electronic continuum in the phonon region. Slakey et al. ${ }^{8}$ working with untwinned crystals find that the electronic Raman scattering is stronger in the chain direction and the coupling to phonons is stronger at 116 $\mathrm{cm}^{-1}$ in the chain direction than normal to it. Nevertheless electronic continuum is present in both directions in the $a b$-plane along with the Fano type coupling to the optic phonons at $q=0$.

It is tempting to identify the $350 \mathrm{~cm}^{-1}$ peak in the Raman continuum with the peak in $\Gamma(\omega)$ since this quantity is according to Eq. 3 proportional to $\operatorname{Im}(1 / \epsilon)$ which in turn enters the expression for the electronic Raman crossec- 
tion. There is a problem with this simple picture however since the dielectric function in the Raman crossection is the total one including the contribution from the condensate whereas the $\Gamma(\omega)$ in Fig. 2 only refers to the midinfrared electronic band.

As in the case of the $350 \mathrm{~cm}^{-1}$ peak in the Raman spectrum the infrared feature at $450 \mathrm{~cm}^{-1}$ as well as the onset at $130 \mathrm{~cm}^{-1}$ have been interpreted as the superconducting gap. $23,24,10$ The difficulties with this interpretation have been pointed out by several groups. ${ }^{11,12}$ In this review we have outlined the evidence for an alternate interpretation of these low lying structures in the infrared spectra of the high $T_{c}$ superconductors in terms of charged phonons interaction with an electronic background.

We thank J.P. Carbotte, H.D. Drew, C. Kallin, M. Reedyk and M.J. Rice for valuable discussions. The research at McMaster is supported by the Natural Science and Engineering Research Council of Canada (NSERC) and the Canadian Institute for Advanced Research (CIAR). Work at Florida is supported by DARPA through contract MDA972-88-J-1006.

\section{REFERENCES}

1. W. Weber and L.F. Mattheiss, Phys. Rev. B 37, 599 (1988).

2. B. Renker, F. Gompf, E. Gering, D. Ewert, H. Rietschek, and A. Dianoux, Z. Phys. B - Condensed Matter 73, 309 (1988).

3. W. Reichard, N. Pyka, L. Pintschovius, B. Hennion, and G. Collin, Int. Conf. on Superconductivity, Stanford, 1989, Physica C. $162-164,464$, (1989).

4. S.L. Cooper, M.V. Klein, B.G. Pazol, J.P. Rice, and D.M. Ginsberg, Phys. Rev. B 37, 5920 (1988).

5. R.M. Mcfarlane, H.J. Rosen, and H. Seki Solid State Comm. 63, 831 (1987).

6. C. Thomsen, M. Cardona, B. Gegenheimer, R. Liu, A. Simon, Phys. Rev. $B$ 37, 9860 (1988).

7. T. Ruf, C. Thomsen, R. Liu, M. Cardona, Phys. Rev. B 3, 11985 (1988).

8. F. Slakey, S.L. Cooper, M.V. Klein, J.P. Price, E.D. Bukowsky, and D.M. Ginsberg, Phys. Rev. B 38, 11934 (1989).

9. For a review of the early work on the infrared properties of the high $T_{c}$ materials see T. Timusk, D.B. Tanner, in Physical Properties of High Temperature Superconductors I, Donald M. Ginsberg, editor, (World Scientific, Singapore, 1989) p. 339.

10. R.T. Collins, Z. Schlesinger, F. Holtzberg, and C. Field, Phys. Rev. Lett. 63, 422 (1989). 
11. S.L. Cooper, G.A. Thomas, J. Orenstein, D.H. Rapkine, M. Capizzi, T. Timusk, A.J. Millis, L.F. Schneemeyer, and J.V. Waszczak, Phys. Rev. $B$ 40, 11358 (1989).

12. K. Kamarás, S.L. Herr, C.D. Porter, N. Tache D.B. Tanner, S. Etemad, T. Venkatesan, E. Chase, A. Inham, X.D. Wu, M.S. Hegde, and B. Dutta, Phys. Rev. Lett. 64, 84 (1990).

13. M.J. Rice, Phys. Rev. Lett. 37, 36 (1976).

14. T.Timusk and D.B. Tanner, Physica $C$ 169, 425 (1990).

15. S. Tajima, S. Uchida, S. Ishibashi, T. Ido, H. Takagi, T. Arima, Y. Tokura, Physica C, 168, 117 (1990).

16. T. Holstein, Phys. Rev 96, 539, (1954); Ann. Phys. (N.Y.) 29, 410, (1964).

17. R.R. Joyce and P.L. Richards, Phys. Rev. Lett. 24, 1007 (1970).

18. W. Lee, D. Rainer, and W. Zimmerman, Physica $C$ 159, 535 (1989).

19. N.E. Bickers, D.J Scalapino, R.T Collins, and Z. Schlesinger, Phys. Rev. $B$ 42, 67 (1990).

20. E. Nicol, J.C. Carbotte, and T. Timusk, Phys. Rev. B (to be published).

21. M.J. Rice, V.M. Yartsev, and C.S. Jacobsen, Phys. Rev. B 21, 3437 (1980).

22. M.J. Rice and Y.R. Wang, Phys. Rev. B 36, 8794 (1987).

23. G.A. Thomas, J. Orenstein, D.H. Rapkine, M. Capizzi, A.J. Millis, R.N. Bhatt, L.F. Schneemeyer, and J.V. Waszczak, Phys. Rev. Lett. 61, 1313 (1988).

24. J. Schützmann, W. Ose, J. Keller, K.F. Renk, B. Roas, L. Schultz, and G. Saemann-Ischenko, Europhys. Lett., 8, 679 (1989).

25. T. Pham, H.D. Drew, S.H. Mosley, and J.Z. Liu, Phys. Rev. B 41, 11681 (1990).

26. M. Reedyk, D.A. Bonn, J.D. Garrett, J.E. Greedan, C.V. Stager, T. Timusk, K. Kamarás, and D.B. Tanner, Phys. Rev. B 38, 11981 (1988).

27. A detailed analysis done in ref. 12 of the optical conductivity above and below the $T_{c}$ shows that the portion of the conductivity that condenses at $T_{c}$ is smooth and Drude-like. The frequency dependent structure is associated with temperature independent midinfrared band. This analysis also demonstrates that the Drude absorption does not develop a gap at $T_{\mathfrak{c}}$.

28. W. Götze and P. Wölfle, Phys. Rev. B 6, 1266 (1972).

29. J.W. Allen and J.C. Mikkelsen, Phys. Rev. B 15, 2952 (1977).

30. G.A. Thomas, M. Capizzi, T. Timusk, S.L. Cooper, J. Orenstein, D. Rapkine, S. Martin, L.F. Schneemeyer, and J.V. Waszczak, J. Opt. Soc. Am. 6, 415,1989 
31. K. Kamarás, C.D. Porter, M.G. Doss, S.L. Herr, D.B. Tanner, D.A. Bonn, J.E. Greedan, A.H. O'Reilly, C.V. Stager, and T. Timusk, Phys. Rev. Lett. 59, 919 (1987).

32. J. Tanaka, K. Kamya, M. Shimizu, M. Simada, C. Tanaka, H. Ozeki, K. Adachi, K. Iwahashi, F. Sato, A. Sawada, S. Iwata, H. Sakuma, and S Uchiyama, Physica C 153-155, 1752 (1988).

33. M.P. Petrov et al. Pis'ma Zh. Eksp. Teor. Fiz., 50, 25, (1989).

34. J. Orenstein, G.A. Thomas, A.J. Millis, S.L. Cooper, D. Rapkine, T. Timusk, L.F. Schneemeyer, and J.V. Waszszak, Phys. Rev. B (to be published).

35. Z. Schlesinger, R.T. Collins, F. Holtzberg, C. Feild, S.H. Blanton, U. Welp, G.W. Crabtree, Y. Fang, and J.Z. Liu, Phys. Rev. Lett. 65, 801 (1990). 